\title{
Endoscopic third ventriculostomy in treatment of hydrocephalus
}

\author{
Paweł Sokal' ${ }^{1}$, Marcin Birski ${ }^{1}$, Marcin Rusinek ${ }^{1}$, Darek Paczkowski ${ }^{1}$, Piotr Zieliński ${ }^{1}$, Aleksandra Harat ${ }^{2}$ \\ 1Department of Neurosurgery, Military Clinical Hospital, Bydgoszcz, Poland \\ 2Institute of Preventive Medicine and Environmental Health, Cathedral of Public Health, Collegium Medicum, Nicolaus Copernicus \\ University, Bydgoszcz, Poland
}

Videosurgery Miniinv 2012; 7 (4): 280-285 DOI: $10.5114 /$ wiitm.2011.30810

\begin{abstract}
Introduction: Endoscopic third ventriculostomy (ETV) is a minimally invasive method of treatment of obstructive hydrocephalus (HCP).

Aim: To investigate perioperative and intraoperative difficulties, failures and complications of ETV.

Material and methods: Seventy-three procedures of ETV were conducted in our department in the last 5 years on 69 patients with HCP of different etiology. In 4 patients we performed ETV twice. In 4 cases we used neuronavigation. In 6 cases ETV was performed in conjunction with endoscopic biopsy of the tumor. In 6 cases we had to repeat the procedure (4) or implant a ventriculo-peritoneal shunt (2) due to recurrence of symptoms.

Results: In our series we had 3 important complications: one thalamic injury and 2 intraventricular hemorrhages. In 4 cases we observed postoperative hyperthermia with the presence of meningeal symptoms. Two cerebrospinal fluid (CSF) leaks were secured with additional stitches and 2 CSF infections were treated with antibiotics. In 1 patient epileptic seizers were observed. Three others complained of nausea and vomiting. The initial success rate of ETV is $70 \%$. Conclusions: Based on our material we conclude that ETV is a useful and helpful procedure in non-communicating HCP. It carries $4 \%$ perioperative risk of serious complications which can be reduced by proper selection of patients, detailed plan and skilful performance of surgery in experienced hands and meticulous postoperative care.
\end{abstract}

Key words: ventriculostomy, endoscope, hydrocephalus.

\section{Introduction}

Hydrocephalus (HCP) is the enlargement of cerebral ventricles, which can be caused by blocked circulation of cerebrospinal fluid (CSF). In communicating HCP the cause of blocked CSF circulation is diminished absorption of CSF in arachnoid granulations, while in non-communicating HCP the obstruction exists proximally to granulations, for instance stenosis of the aqueduct of Sylvius. Obstruction also occurs in neoplastic diseases - tumors closing the lumen of the fourth ventricle and aqueduct of Sylvius [1].
Endoscopic third ventriculostomy (ETV) is a minimally invasive method of treatment of obstructive hydrocephalus (HCP). The ETV opens the circulation of CSF from the third ventricle to subarachnoid spaces, bypassing the obstructed aqueduct or fourth ventricle. In consequence ETV diminishes the size of the lateral and the third ventricles, thus decreasing the intracranial pressure (ICP). The third ventricle should be sufficiently enlarged to allow safe, limited movement of the endoscope without injury to the lateral walls of the ventricle [2]. In management of HCP possible options are ventriculoperitoneal shunts or ETV [1]. The advantage of ETV is low risk

Address for correspondence:

Paweł Sokal MD, Department of Neurosurgery, Military Clinical Hospital, 5 Powstancow Warszawy St, 85-681 Bydgoszcz, Poland, phone: +4852378 70 93, fax: +4852378 70 94, e-mail: psokal@wp.pl 
of long-term complications associated mostly with device malfunction or infections caused by the presence of a foreign object [2].

\section{Indications for ventriculostomy}

Obstructive HCP caused by anatomic, inflammatory or neoplastic etiology is the most common indication [1, 2]. In adults we usually encounter non-communicating HCP caused by tumors. Thalamic, tectal and pineal tumors are not always amenable to resection, and ETV in conjunction with biopsy of that tumor remains the only possible intervention [3-5]. Colloid cysts can be resected endoscopically. Lack of effects after shunting or infectious complications associated with ventriculo-peritoneal shunts may be indications for ETV. Some centers also recommend it in long-standing overt ventriculomegaly (LOVA), Chari malformation and normal pressure hydrocephalus (NPH), although traditionally communicating hydrocephalus has been supposed as a contraindication to ETV $[1,6]$. There is evidence that ETV may be successful in some NPH patients. Possible complications of ETV are hypothalamic injury, basilar artery injury, intracerebral, intraventricular or subdural hemorrhage, CSF leakage, CNS infection, subdural hygroma or hematoma, epilepsy, and headache due to collection of air subdurally over the frontal cortex, with an overall complication rate of $8.5 \%[1,2,7,8]$.

\section{Aim}

In our study we investigated perioperative and intraoperative difficulties, failures and complications based on 73 procedures of ETV conducted in our department in last 5 years.

\section{Material and methods}

Our material consisted of 69 patients with hydrocephalus of different etiology aged 18-74 years, who after written consent were qualified for ETV in the Department of Neurosurgery of the Military Clinical Hospital in Bydgoszcz in the years 2006-2011. In 4 patients we performed ETV twice. Sixty-four patients had non-communicating HCP, and 3 had NPH with symptoms of Hakim syndrome. Patients were operated in general anesthesia. The procedure was performed with the use of an intraventricular neuroendoscopic system made by Aesculap (Tuttlingen, Germany). The system was composed of a trocar containing 4 channels: working, scope, irrigation and overflow. Our endoscope had a direction of view of $30^{\circ}$. In 4 cases we used neuronavigation. In 6 cases ETV was performed in conjunction with endoscopic biopsy of the tumor located usually in the $3^{\text {rd }}$ ventricle or in the close vicinity of this ventricle. Outcome was assessed in the immediate postoperative period based on neurologic status and radiologic examination - head computed tomography (CT). Success of ETV was defined as partial or complete relief of symptoms and decreased size of ventricles. Failure was defined as no change or deterioration in condition.

\section{Endoscopic third ventriculostomy surgical technique}

The ETV procedure begins from the skin incision usually in the right frontal area and burr hole $2-3 \mathrm{~cm}$ lateral to the midline, $1-2 \mathrm{~cm}$ anterior to the coronary suture. A rigid endoscope is passed through the frontal lobe into the lateral ventricle, then through the foramen of Monro to the floor of the third ventricle which is formed by the terminal membrane containing the Liliequist membrane. The goal of the procedure is to make a hole in this membrane anterior to the mammillary bodies and posterior to the infundibular recess. The opening in the membrane is made using a Fogarty catheter no. 2 or 3 , or by another rigid instrument to puncture the floor of the ventricle, then the balloon serves for enlargement of the perforation. Endoscopic visualization enables inspection of structures behind the membrane such as basilar and posterior cerebral arteries. Frequently fast, vibrant flow of CSF through the stoma can be seen. Successful perforation is determined by many factors, including anatomical factors such as narrowed foramen of Monro with oversized choroid plexus lying in the posterior part of the foramen, small triangle made by mammillary bodies and infundibular recess which is the target of perforation, and thick, opaque membrane of the bottom of the third ventricle, which should be perforated. Visualization during the procedure can be worsened by bleeding. Meticulous hemostasis can be achieved by microcoagulation, or long-lasting, excessive washing with warm Ringer solution. Bloody or blurry CSF requires washing, which improves transparency and prevents complications such as thalamus injury, forniceal columns injury, or basilar artery injury. In order to decrease 


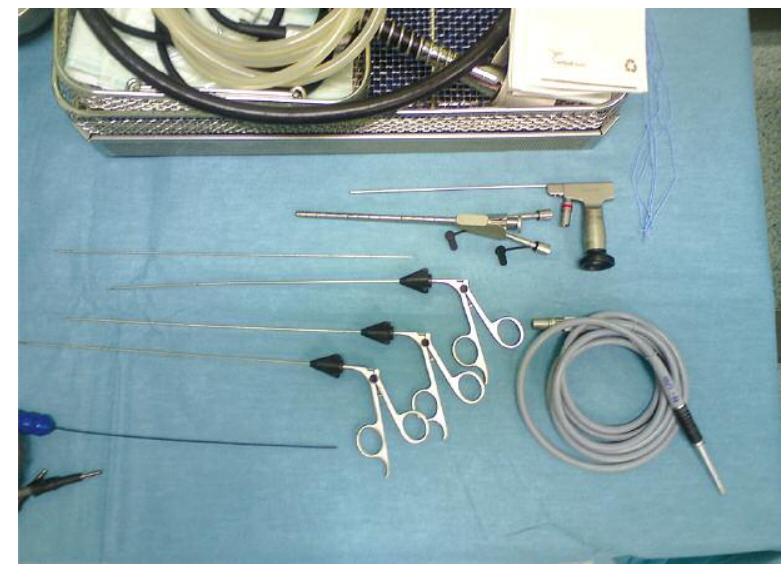

Figure 1. Rigid endoscope set (Minop) for ETV

the risk of perioperative complications magnetic resonance imaging (MRI) is necessary and especially mid-sagittal images enabling optimal localization of the burr hole. It is possible to use neuronavigation to select the most appropriate trajectory from the entry point on the skull to the floor of the third ventricle to avoid straining of the foramen Monro and find the safest, straight way with proper visualization of all structures in the floor.

\section{Results}

We observed a decrease in size in lateral and third ventricles and alleviation of symptoms of increased ICP after performing ETV in 48 patients (whole group 69) with non-communicating HCP based on radiological and clinical examination the day or 3 days after the surgery documented by clinical examination and postoperative CT. In the majority of patients with $\mathrm{NPH}$ we noted clinical improvement in gait and in uri-

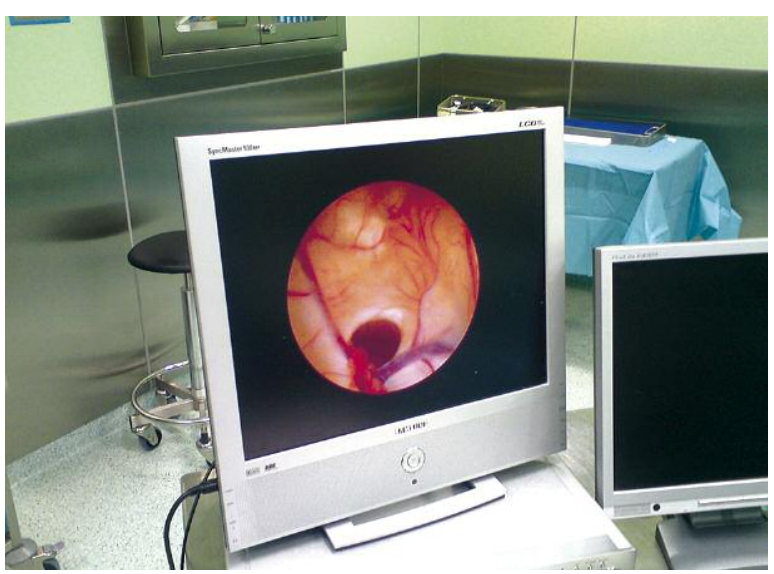

Figure 3. Foramen of Monro visualized on screen

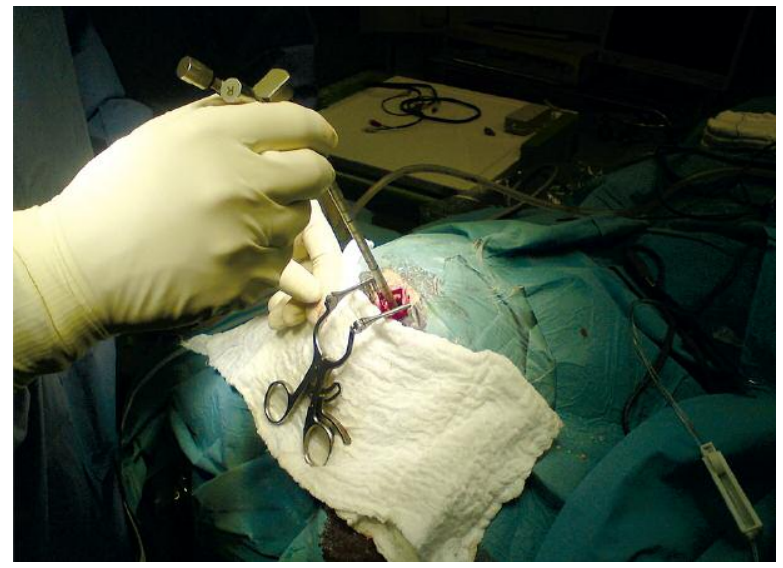

Figure 2. Endoscope in the burr hole

nary sphincter control. In 6 cases we had to repeat the procedure (4) or implant a ventriculo-peritoneal shunt (2) due to recurrence of symptoms: usually headaches, disturbances in gait and speech in different periods after the first ETV. In our series we had 3 important complications: 1 thalamic injury and 2 intraventricular hemorrhages, which gives a $4 \%$ perioperative risk of serious complications. Two CSF leaks were secured with additional stitches and two CSF infections treated with antibiotics. In 1 patient epileptic seizures were observed. Three others complained of nausea and vomiting. In 4 cases we observed postoperative hyperthermia with the presence of meningeal symptoms. There were no procedure-related deaths (Figures 1-11).

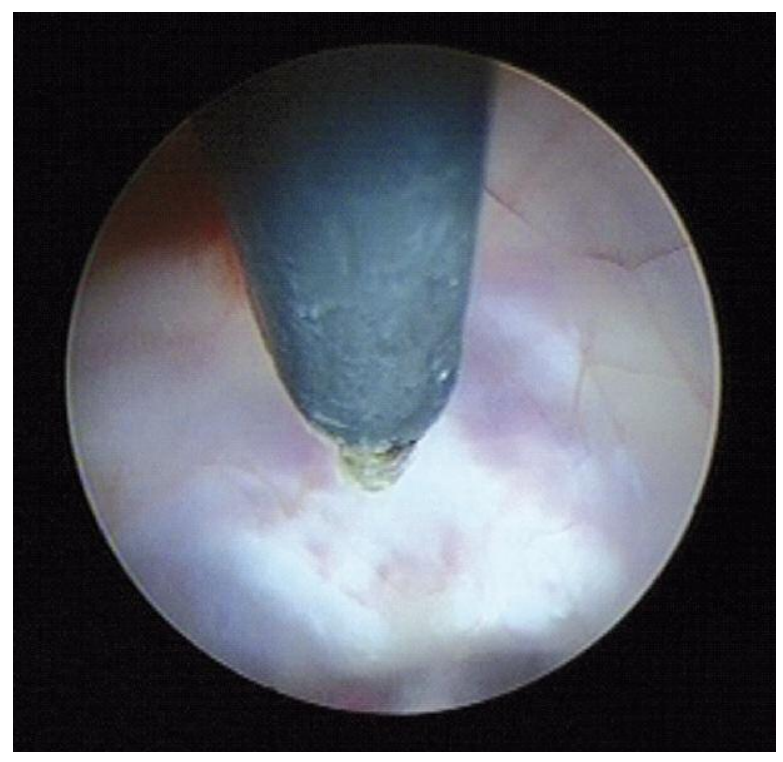

Figure 4. Floor of the third ventricle 


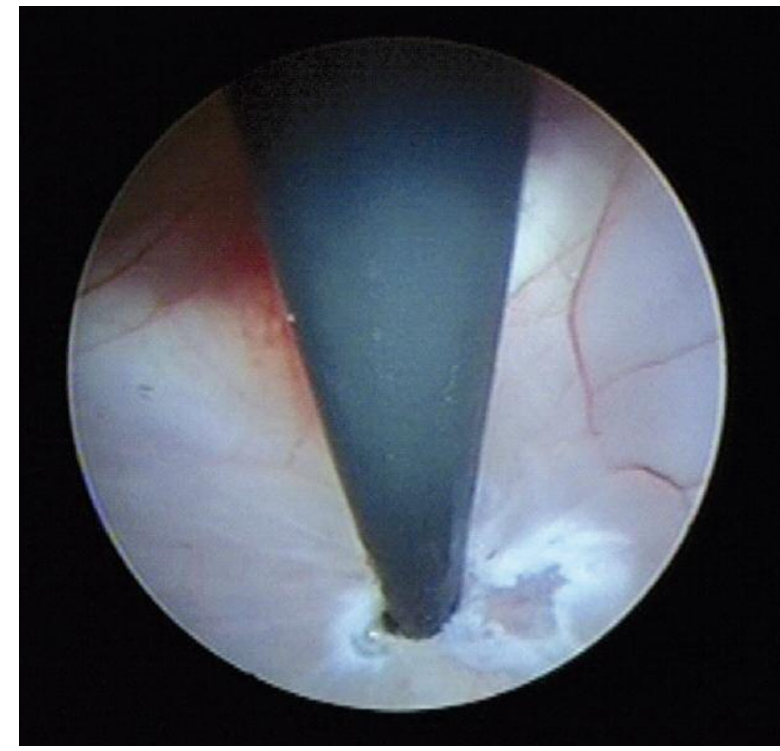

Figure 5. Coagulation of the membrane

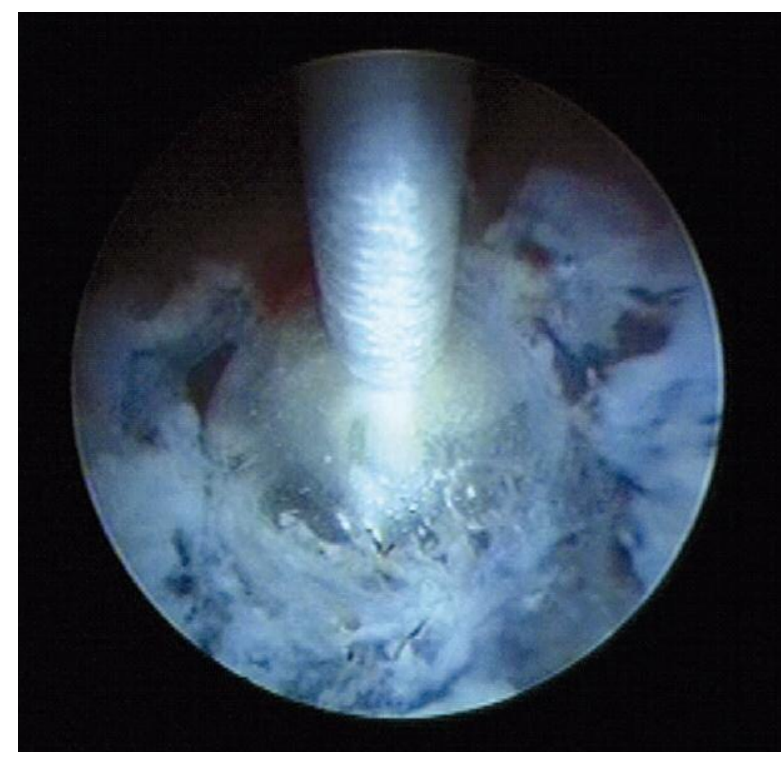

Figure 7. Enlargement of the opening with the balloon

\section{Discussion}

We perform ETV in patients with non-communicating HCP caused by obstruction existing distal to the third ventricle in order to bypass disturbed flow of CSF, which leads to the lowering of ICP. In our neurosurgical practice we very seldom deal with patients with congenital aqueductal stenosis. Frequently we can suspect obstruction based on a picture of triventricular HCP and cine-MRI scans demonstrating the

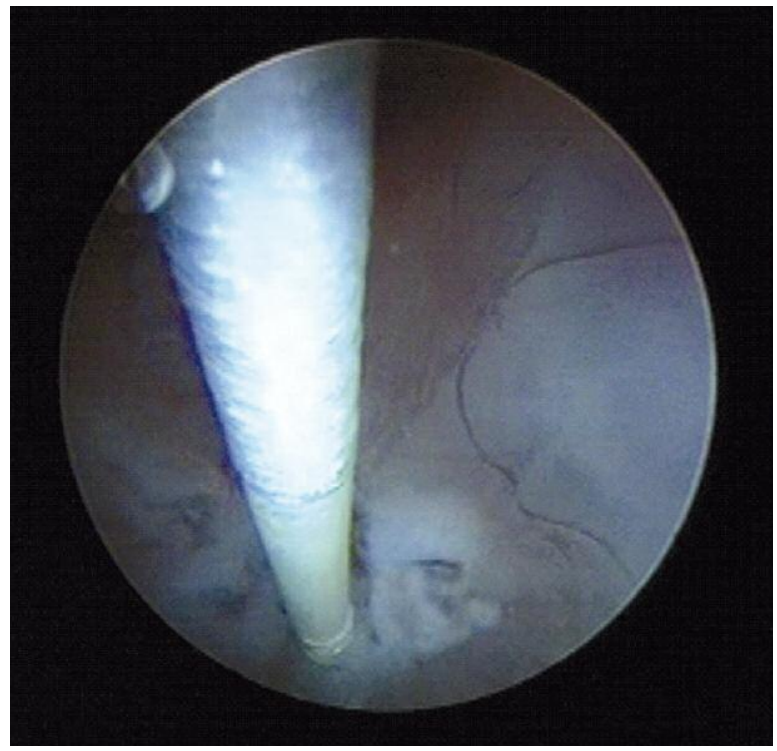

Figure 6. Fogarty catheter in the opening

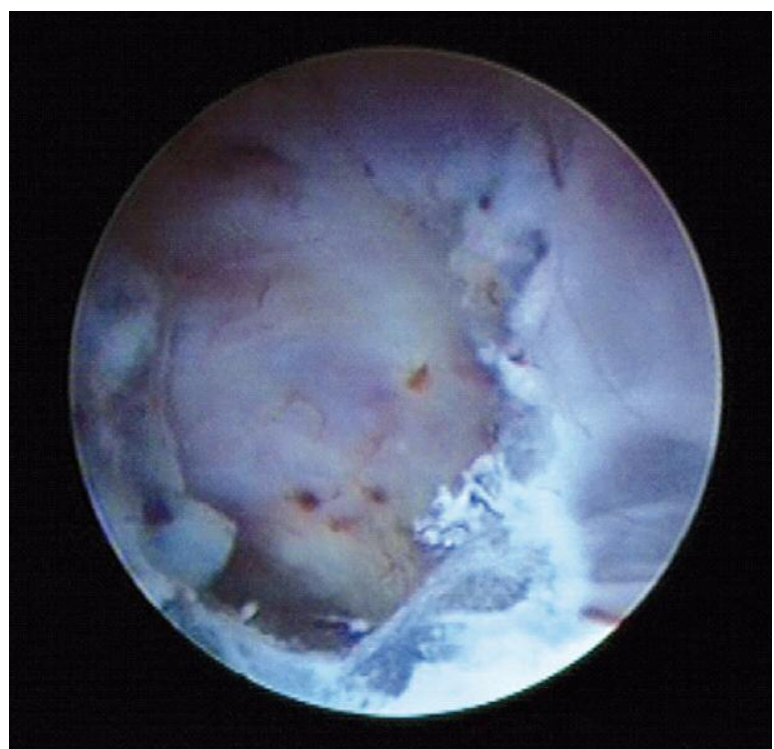

Figure 8. Perforation

flow void phenomenon [9]. Most often we treat patients with tumors obstructing proper flow of CSF. In cases of non-operable tumors, it is the only intervention we conduct. In 6 cases it was possible to combine ETV with biopsy of the tumor, but usually biopsy worsens the conditions of ETV because it causes bleeding which needs irrigation. All patients with triventricular hydrocephalus with sufficient dilatation of the third ventricle are amenable to ETV. The ETV is a minimally invasive procedure, is safe and is espe- 


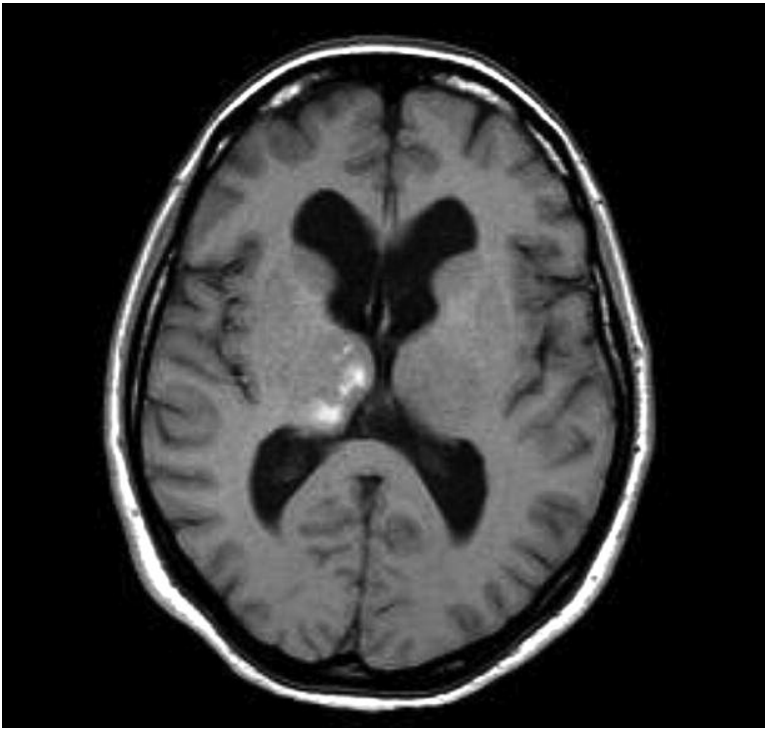

Figure 9. The MRI scans showing thalamic infarct after ETV

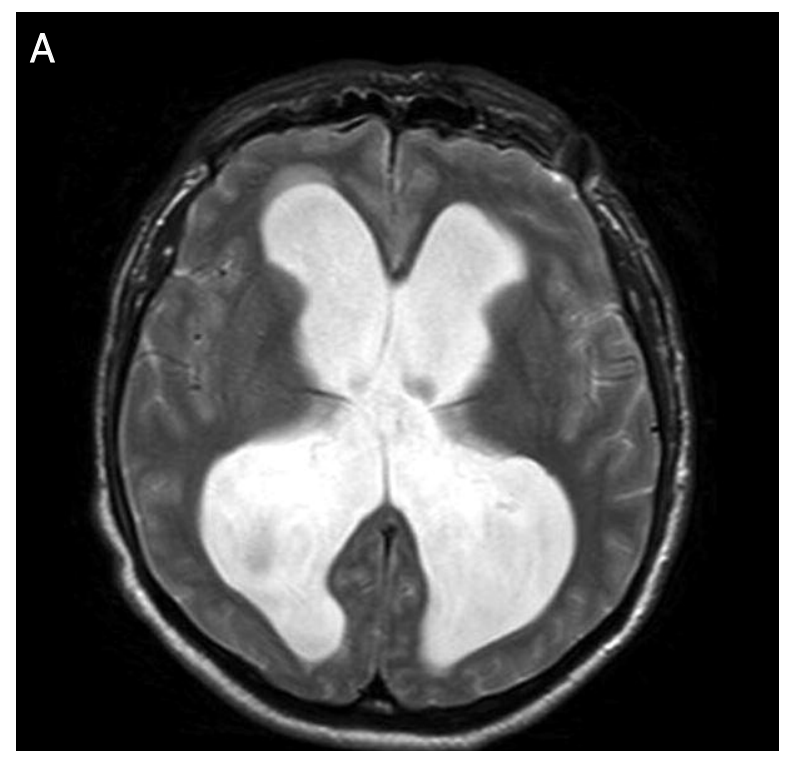

Figure $11 \mathrm{~A}-\mathrm{B}$. Huge triventricular hydrocephalus

cially effective and useful in non-communicating HCP $[1-4,6,7,10,11]$. Alleviation or diminishment of symptoms associated with increased ICP occurred in patients with idiopathic stenosis of the aqueduct in 6 cases in our group, and obstructive HCP caused by tumor in 32 cases. It is also helpful in selected cases of $\mathrm{NPH}$. We noted however that improvement in $\mathrm{NPH}$ cases seems to be not as good as one might expect after ventriculoperitoneal shunt. It is important to select patients for ETV. Patients with a history

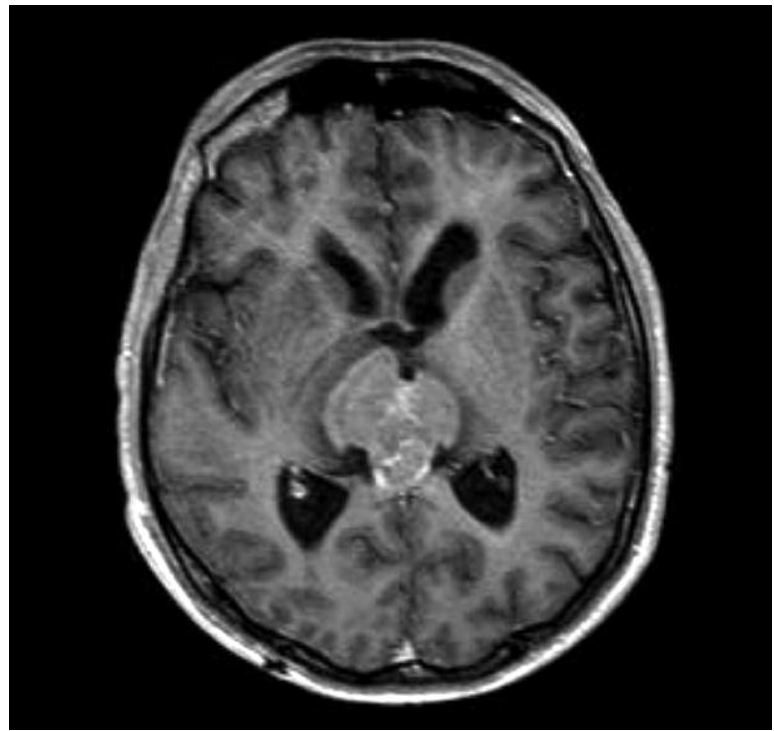

Figure 10. Tumor of the third ventricle qualified for endoscopic biopsy

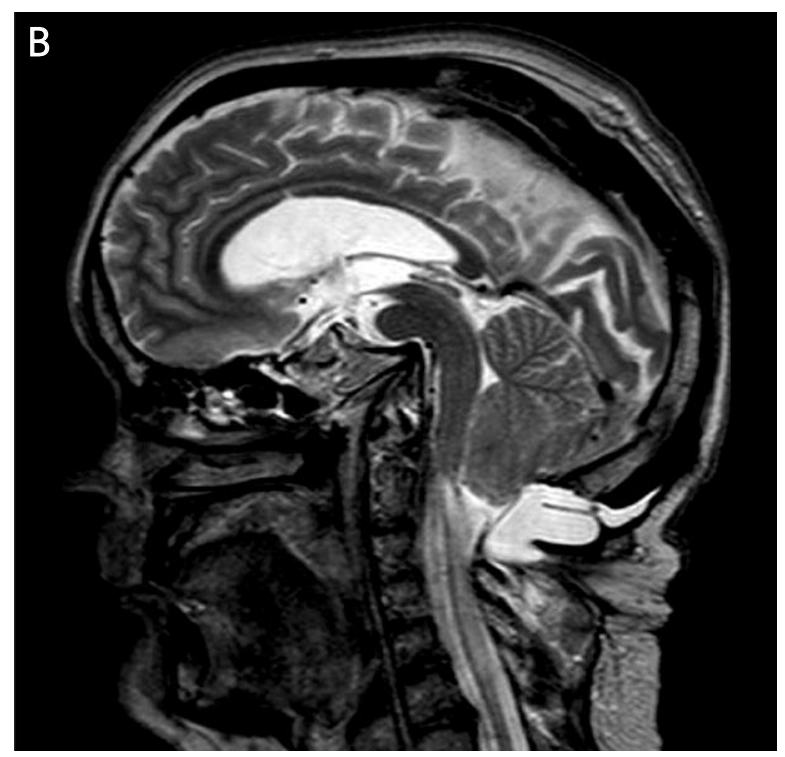

of ventricular hemorrhage, meningitis and leptomeningeal metastasis should be excluded for consideration of ETV because of concern for decreased CSF resorption capacity. Patients with headache associated with nausea, vomiting, or lethargy were more likely to respond to treatment with ETV relative to patients presenting with headache [3]. The success rate of ETV in adults depends on the etiology of HCP and is calculated as $69 \%$ to $83 \%$ [6]. In our group we estimate the initial success rate as around $70 \%$. 
The long-term rate is much lower due to progression of neoplastic disease or reclosure of the stoma.

The ETV is a relatively safe procedure in experienced hands but carries the risk of complications such as hypothalamic or thalamic injury [6]. The technique can be augmented by the use of neuronavigation or stereotactic guidance $[2,4]$. The most common difficulties associated with the surgery are narrow space between mammillary bodies and dorsum sellae, high localization of basal artery bifurcation, and opaque floor of the third ventricle bulging to the sellae [12]. According to Bouras, who conducted a review on complications of ETV, the overall complication rate was $8.8 \%$. Permanent morbidity was $2.1 \%$, neurologic in $1.2 \%$ (hemiparesis, gaze palsy, memory disorders, and/or altered consciousness), hypothalamic in $0.9 \%$ (diabetes insipidus, weight gain, or precocious puberty). Intraoperative hemorrhage was present in $3.9 \%$, severe in $0.6 \%$ (including 4 cases $(0.14 \%)$ of basilar rupture). Other surgical complications occurred in 1.13\% (3 thalamic infarcts, 6 subdural, 6 intracerebral, and 2 epidural hematomas). Cerebrospinal fluid infections occurred in $1.8 \%$, CSF leak in $1.7 \%$, anesthetic complications (bradycardia and hypotension) in $0.19 \%$ of cases [7]. In our series we had 3 important complications: 1 thalamic injury and 2 intraventricular hemorrhages. The CSF leaks can be secured with additional stitches and CSF infections must be treated with antibiotics. Neuronavigation was used in order to set the proper entry point and further to find the optimal trajectory to the floor of the third ventricle and to avoid injury of forniceal columns when passing a rigid endoscope through the foramen of Monro.

In 4 cases of repeated ETV we noticed obliteration of the previously performed foramen in 3 cases with a median of 7 months. Too small foramen in the membrane is more amenable for obliteration in our opinion. A large perforation may prolong the period without symptoms of non-communicating HCP.

\section{Conclusions}

Based on our material we conclude that ETV is a useful and helpful procedure in non-communicating HCP. It carries relatively low perioperative risk of complications (comparable to results presented in the literature), which can be reduced by proper selection of patients, a detailed plan and skilful performance in experienced hands, and meticulous postoperative care of the patient.

\section{References}

1. Greenberg MS. Handbook of Neurosurgery. $6^{\text {th }}$ ed. Thieme, New York 2006; 180-207.

2. Hellwig D, Grotenhuis JA, Tirakotai W, et al. Endoscopic third ventriculostomy for obstructive hydrocephalus. Neurosurg Rev 2005; 28: 1-34.

3. Chen CC, Kasper E, Warnke P. Palliative stereotactic-endoscopic third ventriculostomy for the treatment of obstructive hydrocephalus from cerebral metastasis. Surg Neurol Int 2011; 2: 76.

4. Chibbaro S, Di Rocco F, Makiese O, et al. Neuroendoscopic management of posterior third ventricle and pineal region tumors: technique, limitation, and possible complication avoidance. Neurosurgical Review, Online First ${ }^{\top M}, 19$ January 2012.

5. Macarthur DC, Buxton N, Punt J, et al. The role of neuroendoscopy in the management of brain tumours. Br J Neurosurg 2002; 16: 465-70.

6. Jenkinson MD, Hayhurst C, Al-Jumaily M, et al. The role of endoscopic third ventriculostomy in adult patients with hydrocephalus. J Neurosurg 2009; 110: 861-6.

7. Bouras T, Sgouros S. Complications of endoscopic third ventriculostomy. A review. J Neurosurg Peditarics 2011; 7: 643-9.

8. Sgaramella E, Castelli G, Sotgiu S. Chronic subdural collection after endoscopic third ventriculostomy. Acta Neurochir 2004; 146: 529-30.

9. Penn RD, Basati S, Sweetman B, et al. Ventricle wall movements and cerebrospinal fluid flow in hydrocephalus. J Neurosurg 2011; 115: 159-64.

10. Kwiek SJ, Mandera M, Bazowski P, et al. Endoscopic third ventriculostomy for hydrocephalus: early and late efficacy in relation to aetiology. Acta Neurochir 2003; 145: 181-4.

11. Stachura K, Libionka W, Moskała M. Neuroendoscopic third ventriculostomy in the management of noncommunicating hydrocephalus secondary to giant basilar artery bifurcation aneurysm - case report and review of literature. Neurol Neurochir Pol 2008; 42: $255-62$

12. Grand W, Leonardo J. Endoscopic third ventriculostomy in adults: a technique for dealing with the neural (opaque) floor. J Neurosurg 2011; 114: 446-53.

Received: 28.03.2012, Revised: 24.06.2012, Accepted: 7.07.2012. 